Original Research Paper

\title{
High-Speed for Data Transmission in GSM Networks Based on Cognitive Radio
}

\author{
${ }^{1}$ Al Smadi Takialddin, ${ }^{2}$ Kalid Al Smadi and ${ }^{3}$ Orayb O. AL-Smadi \\ ${ }^{1,3}$ Department of Communications and Electronics Engineering, \\ College of Engineering, Jerash University, 311, Jerash-Jordan \\ ${ }^{2}$ Jordanian Sudanese Colleges for Science and Technology, Khartoum, Sudan
}

Article history

Received: 06-01-2017

Revised: 07-01-2017

Accepted: 02-02-2017

Corresponding author:

Al Smadi Takialddin

Department of

Communications and

Electronics Engineering,

College of Engineering, Jerash

University, 311, Jerash-Jordan

Email: takialddina@yahoo.com

\begin{abstract}
This paper mainly studies data transmission DT rate in the modern communication systems. Increasing the need to improve the sensitivity of the control systems to detect low signals weak capacity density in view of the increasing communications systems new radio that use the capacity is decreasing and the scope of the taking of spaciousness and frequencies are on the rise. This proposed approaches Methodology: Of the combined macro cellular and micro cellular networks on the principles of the cognitive radio, Provide in parallel with the work of the main GSM network to transfer data at a rate of several tens of megabits per second depending on the High-speed for Data transmission in GSM networks based on Cognitive Radio (CR) technology and its various aspects that may play a very important role in the field of next generation wireless communication networks.
\end{abstract}

Keywords: Mobile and Wireless Technologies, Cellular Communication, Data Transmission, GSM, Cognitive Radio

\section{Introduction}

Wireless communication is the fastest growing sector of the communication industry. In this exact sense, it has captured the attention of everyone in the whole communications market. Analysis showed that cellular systems are constantly growing year round and presently there are about two billion mobile users all over the world. In point of fact, cellular mobiles have grown into an important business device and part of everyday life for common people. Moreover, wireless communication has replaced many wired line networks in domestic as well as professional life. Many types of equipment are remotely accessible and controlled with the help of wireless communication since radio frequency is the only spectrum which enables wireless.

Communication between transmitter and receiver and because of the increasing number of users within the Radio frequency spectrum, it's not possible to add extra Users. We have already adopted lots of technique to reduce the size of transmitting packet size. In $3 \mathrm{G}$ we adopted Time Division Multiplexing (TDM) and Frequency Division Multiplexing (FDM), but this brings.
Congestion on when the number of users is high. Code Division Multiple Access (CDMA) was the first multiple access technology adopted for $3 \mathrm{G}$ where the information was shared between multiple users using a single channel and it also provided one of the most secure communications (Mei Akyildiz et al., 2009). The Consistent growth of wireless appliances (Wi-Fi) gives the clustered Industry, Scientific and Medical (ISM) bands. People are not able to utilize the spectrum properly due to the growth in ISM bands which leads to a demand for an additional spectrum for good Quality of Service (QoS). Multiple-carrier transmission is one of the most advanced techniques used in many recent applications; the concept behind this technique is to divide one carrier into a number of multiple-subcarriers. The available bandwidth is subdivided into smaller bands with limited bandwidth, each subcarrier having a smaller bandwidth compared to the single carrier system. Each symbol is modulated with each carrier and transmitted through antenna to the channel and received signal is demodulated by the demodulators. Implementing a bank of Modulators and Demodulators is challenging and practically it is not possible. The efforts to standardize the parameters associated with 
this technology found their way in 3GPP Release 9. The ITU contains the document "Energy costs reduction with implementation of "Capacity Transfer Cellular Network" for rural and remote areas" which determines a substantial saving of capital and operating costs during the construction of GSM networks (Mei Akyildiz et al., 2009; Akyildiz et al., 2006).

The main reason for limiting the prospects of GSM usage is relatively low data rates in Radio Access Network (RAN).

The concept of 3GPP cellular communication development presupposes that the future RAN network should be "smart" and include elements of adaptation according to the spectrum and level of radiation and also it should provide a dynamic spectrum allocation, higher values of throughput and power efficiency, active resources sharing The concept of the cognitive radio meets these requirements.

\section{Research Problems}

The existing GSM networks almost fully meet the user's requirements for voice services, but the data transfer rates cannot compete with the cellular networks based technologies. The spectrum wireless bandwidth is shared resources between all participants it is necessary to find a way to divide the breadth between more than a number of participants. It was therefore the selection of the method of integration between multiple routes frequency division FDMA time division of VSAT where part includes bandwidth DIVISION The Division breadth presented by $25 \mathrm{MHz}$ to 124 , holding a signboard with (carrier) away from some of them by 200 $\mathrm{kHz}$. Then divided each of these frequencies carrier time-division in itself the use of the VSAT where the timetable for the statute in time-division equals $15 / 26 \mathrm{~ms}$ about $577 \mu$ s this is called the basic part burst.

The Group bursts with some of the so-called and eight times burst per any $120 / 26 \mathrm{~ms}$ or the equivalent of $4.615 \mathrm{~ms}$. This column is the basic part logical channel the term burst per represent the physical channel per through the column, known as the channel according to the number and placement of bursts.

The Channel encryption ensures protection and privacy in the faculties of the reception and transmission.

In the system of the GSM enter speak, is composed of the parts that represent the samples encrypted talk for $20 \mathrm{~ms}$ each sample. The first part has a 182 bits is the most important and Part II contains 78 bits. Encryption is the first part by adding 4bits are frequently spotted and 3 bit parity. Then encrypts the gross and $\$ 189 \mathrm{mln}$ in offering bits by the encoding the rate of $1 / 2$ as gross 387 bits add again the second part 78 bits, which did not encrypts. As the final product of 456 bits represents a sample talk period $20 \mathrm{~ms}$.
The expansion of bandwidth for the new mobile communication systems has become possible thanks to a shift to the higher radio frequency ranges. However, due to physical constraints the radio waves propagation distance on higher frequencies reduces (Anis et al., 2010) (Table 1) and results in the increase in:

- Number of Base Stations (BS) and trunks between the BS and controller

- Number of handovers at a Subscriber Station (SS) movement, which reduces the probability of successful call completion

- Costs of cellular network resources for connection, handover and alarm processes control in case of a subscriber movement within the communication area with small cell sizes

The frequency resource deficiency results in the cellular network limitation in terms of data transfer rate.

The data transfer rate in the modern communication systems is increased also due to the usage of high-level signal modulations requiring greater signal/noise ratio at the receiver input. In this regard, the claimed characteristics of UMTS and LTE systems in terms of the data transfer rate can be achieved only at a small distance from the BS. In these circumstances, the task of searching and investigating new ways of cellular networks building in order to provide high data rates is of immediate interest at the moment and, first of all, it refers to GSM, the most common standard for cellular communications.

\section{Principles of Cognitive Radio}

In cellular communication, the user requirements are increased continuously. The evolution in wireless technologies explores future mobile systems. The four main aspects of cellular are radio access, bandwidth, data rates and switching schemes. First generation cellular system is an analogy system, providing a bandwidth from 10 to $30 \mathrm{KHz}$ and the data rates ranges are around $10 \mathrm{Kbps}$. The Second Generation (2G) is a GSM system, its bandwidth ranges around $200 \mathrm{KHz}$ and its data rates ranges up to $50 \mathrm{Kbps}$. Third Generations $(3 \mathrm{G})$ provides a bandwidth of $5 \mathrm{MHz}$ and its data rates ranges up to 50 Mbps and 4G cellular system provides a variable bandwidth of 20, 40 and $70 \mathrm{MHz}$ and its data rates from $100 \mathrm{Mbps}$ and to $1 \mathrm{Gbps}$ at the downlink. The Fourth Generation (4G) is an advance cellular system which includes generally LTE advanced and WIMAX advanced systems. Cognitive radio is a Fifth Generation (5G) system; it is a new way of using the spectrum. There are new frequency bands and wider spectral bandwidth per frequency channel. For example, in some areas where broadcast spectrums are not used, 
spectrum can be utilized for mobile communication by cognitive radio technology. It is able to utilize the free portion of licensed spectrum with unlicensed users. It adapts to wireless environments (Chen et al., 2010; Dashti et al., 2012). Cognitive Radio with Multicarrier Technology Multiple carrier technique is one of the most advanced techniques used in many recent applications. The concept behind this technique is to divide one carrier into a number of multiple subcarriers. The available bandwidth is the subdivided into small limited bandwidths, each subcarrier having a smaller bandwidth compared to the single carrier system (Baldini et al., 2010; Benedetto et al., 2008). Each Symbol is modulated with each carrier and transmitted through antennas to the channel and then the received signal is demodulated by the demodulators. A transmitter block diagram of MCM is shown below in Fig. 1.

The input symbols are passed to the serial to parallel converter because we transmit $\mathrm{N}$ symbols in parallel and $\mathrm{i}^{\text {th }}$ data stream is modulated onto $\mathrm{i}^{\text {th }}$ subcarrier. After that, the sum all the subcarriers form a composite signal to transmit on a channel. At the receiver, the composite signal is amplified and passed to the bank of demodulators and then is converted from parallel to serial to symbol stream form. A receiver block diagram of an MCM is shown below in Fig. 2.

MCM transmits $\mathrm{N}$ symbols using $\mathrm{N}$ subcarriers in time period N/B, where B is the Bandwidth (Symbol rate $=\mathrm{N} /(\mathrm{N} / \mathrm{B})=\mathrm{B})$. So that the overall symbol rate in single carrier Vs multicarrier systems remains unchanged. The wideband system divides into narrowband subcarriers in parallel, but the overall data rate remains unchanged, however, implementation wise, it gives significant advantage, because its Narrowband subcarriers experience frequency flat fading and bandwidth becomes less coherent. Hence it is far easier to implement in reliable detection schemes when the receiver and Inter Symbol Interference (ISI) are low. This is the essential idea behind the Multicarrier Modulation (MCM). Implementing a Bank of Modulators and Demodulators is challenging and it's not possible in practice. Instead of using N-Modulator and demodulators in MCM, which can be simply generated by IFFT operation, known as OFDM, This is how fast operation is implemented on a DSP chips in a modern communication systems.

Table 1. The number of frequency channels available in GSM-900, GSM-1800 and E-GSM in case of work of three operators

\begin{tabular}{llllll}
\multicolumn{6}{c}{ Table 1. The number of frequency channels available in GSM-900, GSM-1800 and E-GSM in case of work of three operators } \\
\hline
\end{tabular}

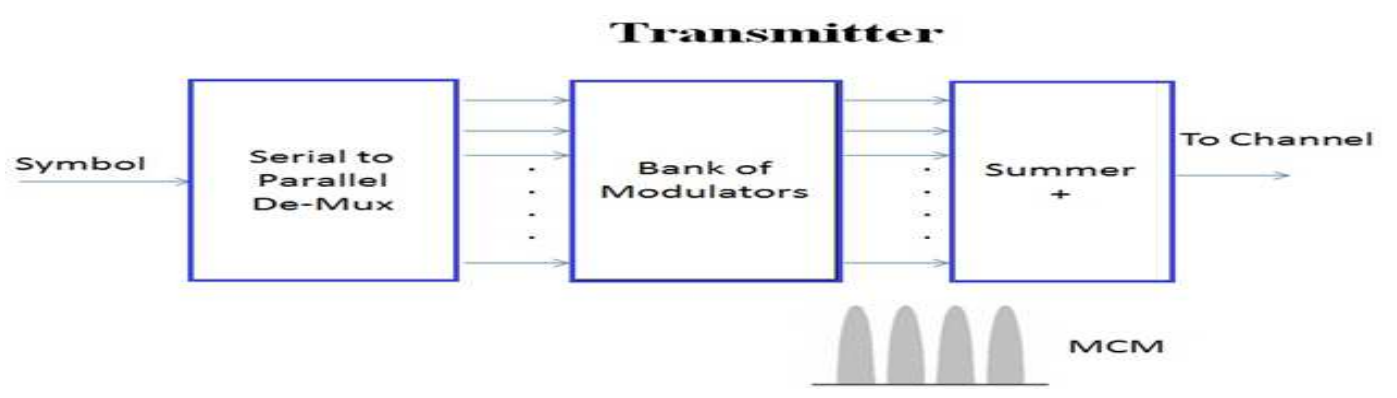

Fig. 1. Block diagram of transmitter

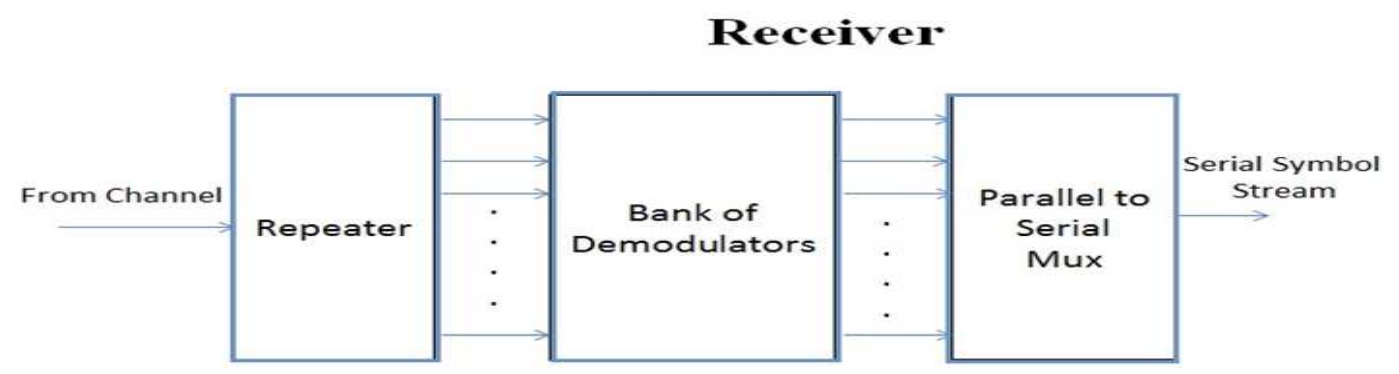

Fig. 2. Block diagram of receiver 


\section{Related Work}

The existing macro-cellular with sizes (1 to $20 \mathrm{~km}$ ) GSM network topology is overlaid with a mutually complementary micro-cellular network operating in the same frequency bands - 900 and/or $1800 \mathrm{MHz}$ (Fig. 1). The macro-cellular network should be denoted as the existing standard GSM network in the frequency bands of 900 and/or $1800 \mathrm{MHz}$, the micro-cellular one is an overlay communication network with cells of smaller sizes $(400-2000 \mathrm{~m})$.

Let us consider the principle of topological combination of the macro- and micro-cellular GSM networks. The existing networks in the area of each specific microcell of the operating GSM network do not use all radio channels provided by the standard, which allows the overlay micro-cellular network to use free GSM frequencies in this area providing the availability of Electromagnetic Compatibility (EMC). An example of shared radio frequency bands $880-915$ and $925-960$ $\mathrm{MHz}$ by macro- and overlay micro-cellular GSM networks is shown in Fig. 2.

To ensure EMC of the networks, the radiation parameters of the base stations of macro- and microcellular GSM networks are selected on the basis of their frequency-and-separation distance that exclude mutual negative influence. The shared elements in both networks are: Main Switching Center (MSC), Home Location and Visitor Location Registers (HLR and VLR), Authentication Center (AuC), Operation and Maintenance Center (OMC). Thus, all functions of authentication, handover, switching and interaction with external networks are performed by infrastructure of the operating GSM network (Fig. 4).

Switching Centre (MSC), Home Location and Visitor Location Registers (HLR and VLR), Authentication centre $(\mathrm{AuC})$, Operation and Maintenance Centre (OMC). Thus, all functions of authentication, handover, switching and interaction with external networks are performed by infrastructure of the operating GSM network (Fig. 3).

\section{Data Transmission}

Maximum data transfer rate of the complementary micro-cellular network when implementing various frequency reuse schemes, There are several models of clusters building with different frequency reuse factors in cells $(K)$ (Ekin et al., 2012; Ng et al., 2016).

The maximum number of frequency channels $\left(N_{\max }\right)$ in GSM-900, GSM-1800 and E-GSM equals to $N_{\max }=N_{G S M-900}+N_{G S M-1800}+N_{E G S M}=124+374+50=$ 548 .

The number of available frequency channels (not used in the cluster) $N_{\text {free }}$ is determined according to the equation":

$$
N_{f e e}=N_{\max }-K N_{f r} N_{o p}\left(N_{f r e e}=N_{\max }-K N_{t s} N_{a g}\right)
$$

The theoretically possible total data transfer rate in the sector $\left(V_{\max }\right)$ with GMSK modulation standard for GSM is One TDMA-frame contains 8 time slots. The maximum data transfer rate in one time slot is 14.4:

$$
V_{\max }=N_{t s} V_{\max t s} N_{\text {free }}
$$

The number of available frequency channels and the maximum data rate in GSM-900, GSM-1800 and E-GSM depend on the frequency reuse factor and the number of frequencies used in the cell of the BS. Table 2 show the data for the most common clustering models typical for work of the three operators in one area that use the same model of clusters building. Particularly, shows that the maximum data rate for three mobile operators at $K=7$, four frequency channels in each cell and simultaneous usage of GSM900, GSM-800 and E-GSM frequency bands is 38 Mbit/s for cell in Table 3.

As shown in Fig. 5 and 6, a spectral sharing has been done between a primary user and two secondary users.

Table 2. Parameters definition

\begin{tabular}{ll}
\hline Name & Definition \\
\hline$N_{\text {free }}$ & The number of free frequency channels \\
$\mathrm{N}_{\text {op }}$ & The number of operators in the area \\
$\mathrm{N}_{\mathrm{ts}}$ & The number of time slots in the TDMA time \\
& frame of the GSM \\
Vmax ts & $\begin{array}{l}\text { Is the maximum data transfer rate in one time } \\
\text { slot of the TDMA-frame of the GSM }\end{array}$ \\
$\mathrm{N}_{\mathrm{f}}$ & The number of frequencies \\
$\mathrm{N}_{\mathrm{ag}}$ & The number of agents
\end{tabular}

Table 3. The maximum data transfer rate (Mbits/s) using GSM-900, GSM-1800 and E-GSM simultaneously in case of three

\begin{tabular}{|c|c|c|c|c|c|c|}
\hline \multicolumn{7}{|c|}{${ }_{f}^{N}$ (Number of frequencies) } \\
\hline$k$ & 1 & 2 & 3 & 4 & 5 & 6 \\
\hline 7 & 56.98 & 48.61 & 43.84 & 39.03 & 34.19 & 29.58 \\
\hline 9 & 54.8 & 45.94 & 39.72 & 33.48 & 28.54 & 25.43 \\
\hline 12 & 50.69 & 41.89 & 33.48 & 27.6 & 23.36 & 19.2 \\
\hline
\end{tabular}
operators 


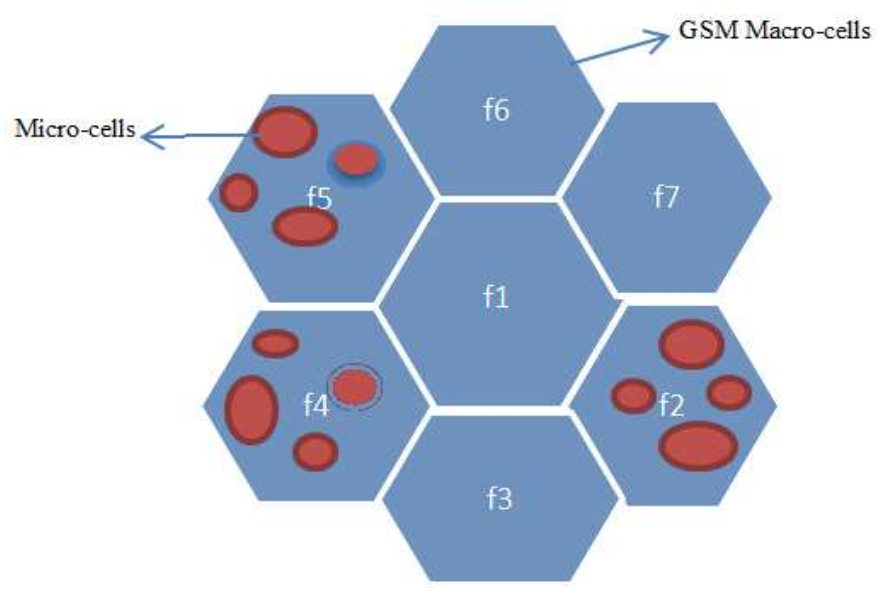

Fig. 3. GSM macro-cellular network overlaid with the micro-cellular network

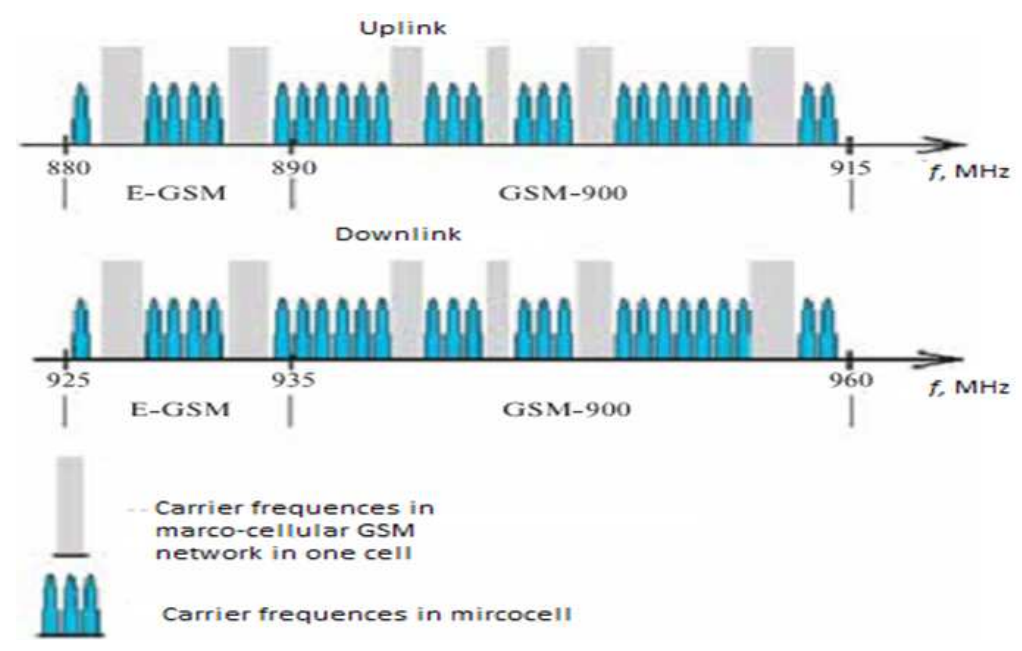

Fig. 4. Example of joint usage of radio frequency bandwidth by macro-cellular GSM network and overlay micro-cellular network

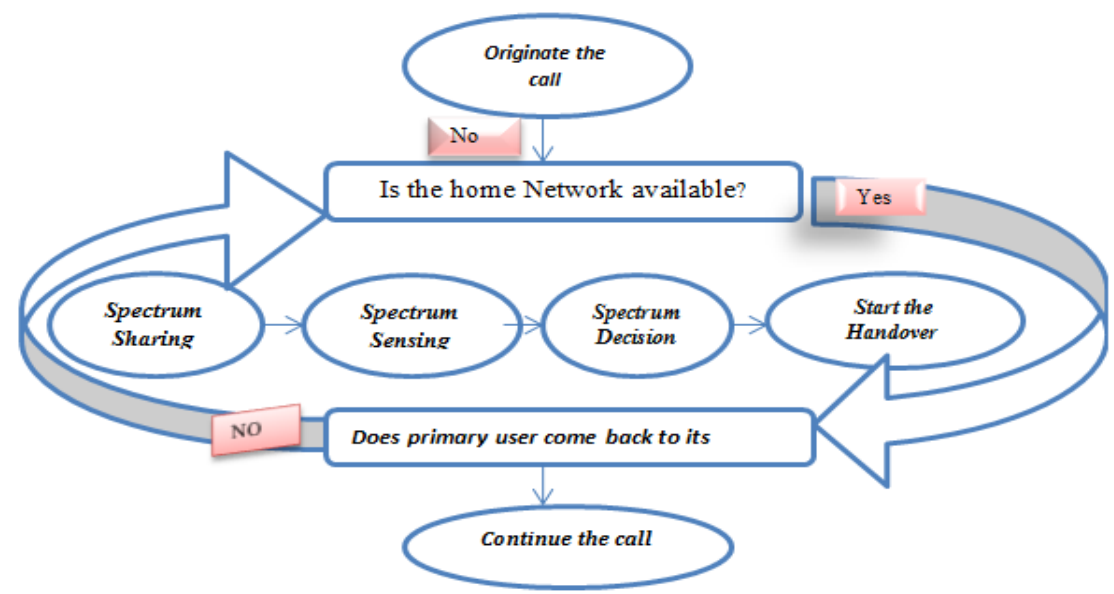

Fig. 5. Shows the Flow chart of this algorithm, as mentioned above this type of DT is an inter-network. Therefore, for the legal issues and the expenditures, it is necessary to use roaming in this DT 


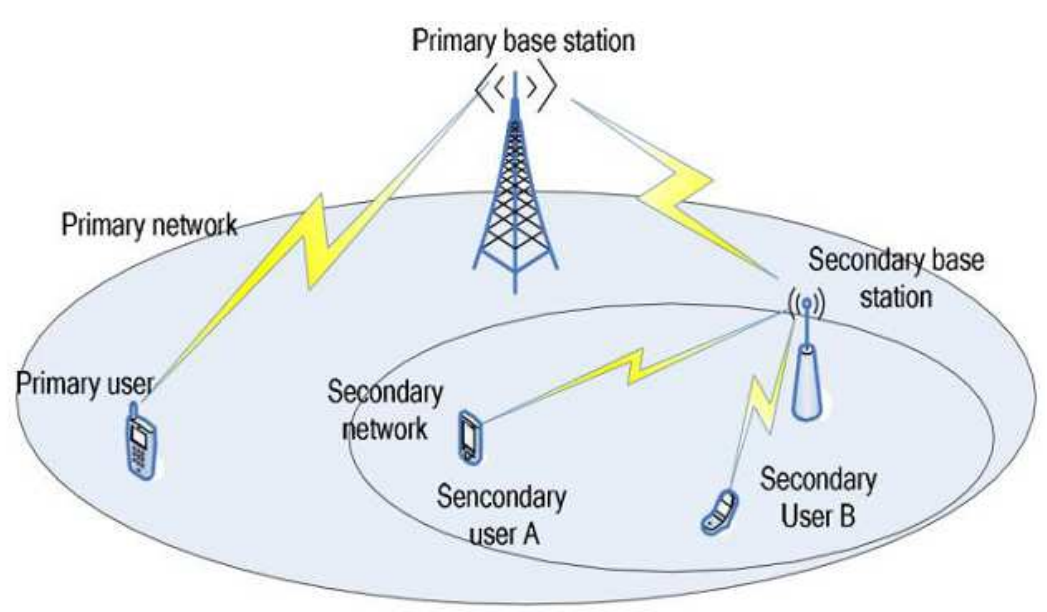

Fig. 6. Spectral sharing between a primary and secondary users

The Adaptive Selection of Operating Frequencies for Micro-Cellular Communication Network

During the operation of a micro-cellular data communication network, the information about unused frequencies comes in the real time mode on the basis of monitoring of the frequencies occupied by the operating GSM network. In general, the frequency resource occupied in a particular point should be evaluated both on the side of the BS and the SS to avoid macrocellular GSM network's interference (Fig. 3). The next step is selection and coordination of available frequencies after which the data transfer process begins in a micro-cellular network.

Since each BS of the micro-cellular network has a smaller coverage area compared to the BS of the macro-cellular GSM network, it can be assumed that distribution of occupied frequencies for the macrocellular GSM network within a microcell is identical. Consequently, there is no need to define protected (occupied) frequencies for the micro-cellular network both at the BS and the subscriber stations. It is enough to have a measuring receiver only at the BS of the microcellular network and translate data on the occupied frequencies within the operating area. This simplifies the design of the $\mathrm{SS}$, releases its computing resources and reduces the amount of routing traffic between the BS and the SS needed to keep the operating frequency coordination within the data transfer process.

If the BS of the micro-cellular network is located within the GSM network coverage area, the operating frequencies of which are known in advance (for example, both networks owned by one operator), there is no need to monitor occupied frequencies at the BS of the micro-cellular network. It becomes possible to program it in advance to exclude radiation of frequencies forbidden in this area, which allow omitting the receiverdetector of occupied channels. Translation of data about occupied frequencies at the SS of the micro-cellular network should take place under the same conditions as in the previous case.

Formation of the OFDM-Signal in the MicroCellular System of Data Transfer with the Exception of Operating Frequencies Occupied by the Macro-Cellular GSM Network

It is advisable to use the OFDM technique for construction of micro-cellular data communication system (Parvin et al., 2012; Stevenson et al., 2009). This contributes to flexible control of the emission bandwidth depending on the number of frequencies occupied by the macro-cellular GSM network at a particular location and will increase the efficiency of data reception in multipath conditions.

In this case the OFDM benefits include: High spectral efficiency due to "almost rectangular frequency spectrum" for a large number of subcarriers; simple digital implementation using a fast Fourier transform; relative simplicity of the receivers; flexible spectrum realization of the OFDM subcarriers rejection by means of programmable methods.

The drawbacks include: A high peak-factor (peak to average power ratio) which requires using a high linearity amplifier; loss in spectral efficiency due to the guard interval with GSM frequencies; great influence on system operation due to the phase noise caused by imperfection of the transmitter and receiver generator; a need for precise frequency and time synchronization.

Exclusion of forbidden frequencies from the common bandwidth of the GSM network in the micro-cellular network occurs as follows. The OFDM symbol is a group of subcarrier frequencies currently transporting the parallel bits of digital streams. The complex envelope of one OFDM-symbol with duration of $T$, which starts at time $t_{k}$, is written as (Khan et al., 2016): 


$$
U(t)=\sum_{i=0}^{N s-1} d_{i} e^{j 2 \pi \frac{i}{t}}\left(t-t_{k}\right) ; t_{k} \leq t \leq t_{k}+T
$$

(d) is a complex number that represents the amplitude and the initial phase of the (i)-th subcarrier of the OFDM-signal; $N_{s}$ is a number of subcarrier oscillations in the OFDM-symbol. The block diagram of formation of the OFDM-symbol's complex envelopes exemplified by the four subcarriers $\left(N_{s}=4\right)$ is shown in Fig. 7 and the corresponding spectral density of the OFDM-signal for $N_{s}=4$ is shown in Fig. 8 .

In case if it is known which GSM frequencies are not able to transfer data in the micro-cellular network, it is necessary to exclude radiation on these frequencies pre-counting the subcarriers that coincide with the forbidden frequencies. To exclude radiation on the $i$-th frequency of the macro-cellular GSM network, according to (1), $d$ must be equated to zero when forming the OFDM-symbol. For example, in case of exclusion of radiation on the 2 nd and $3 \mathrm{rd}$ frequencies, $d=d=0$ (Fig. 9).

The original diagram should be changed by setting the zero symbols at predetermined positions in order to generate OFDM-symbols with the possibility of frequencies exclusion. This can be done during a serialto-parallel conversion of the QAM-symbols stream if the numbers of exclusive frequencies are known. Figure 10 shows the changed diagram of formation of the OFDM symbols' complex envelopes with the exception of occupied frequencies (in this case, the 2 nd and 3rd).

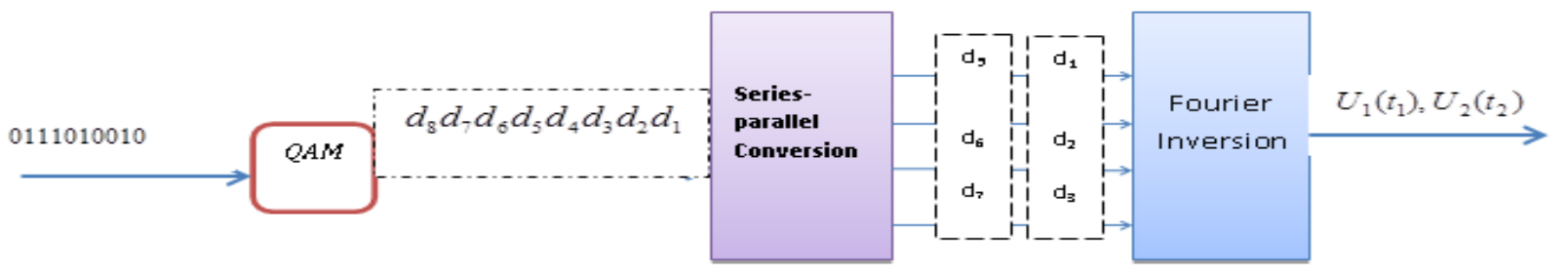

Fig. 7. Block diagram of the OFDM-symbol's complex envelopes forming

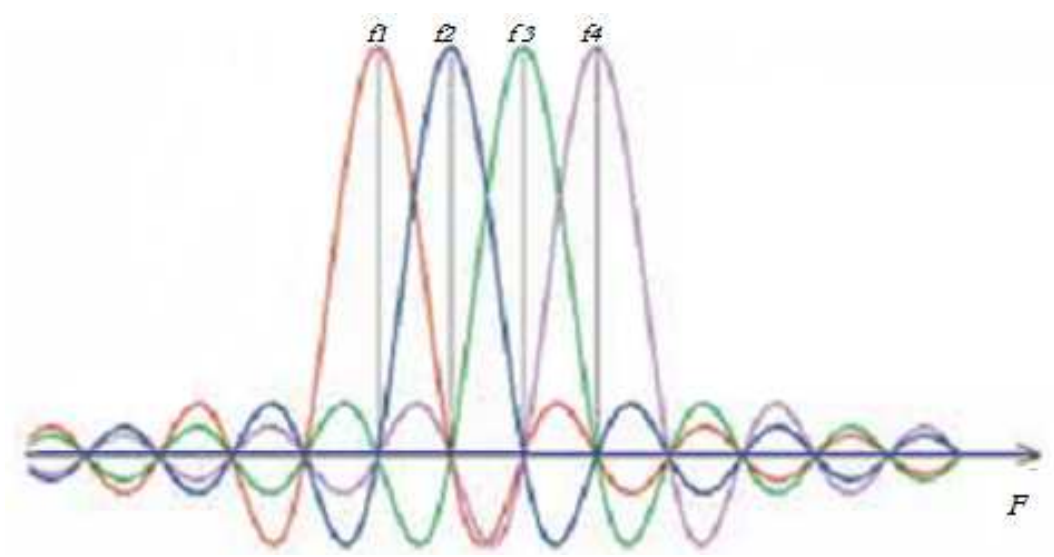

Fig. 8. Spectrum density of the OFDM-signal for $N_{s}=4$

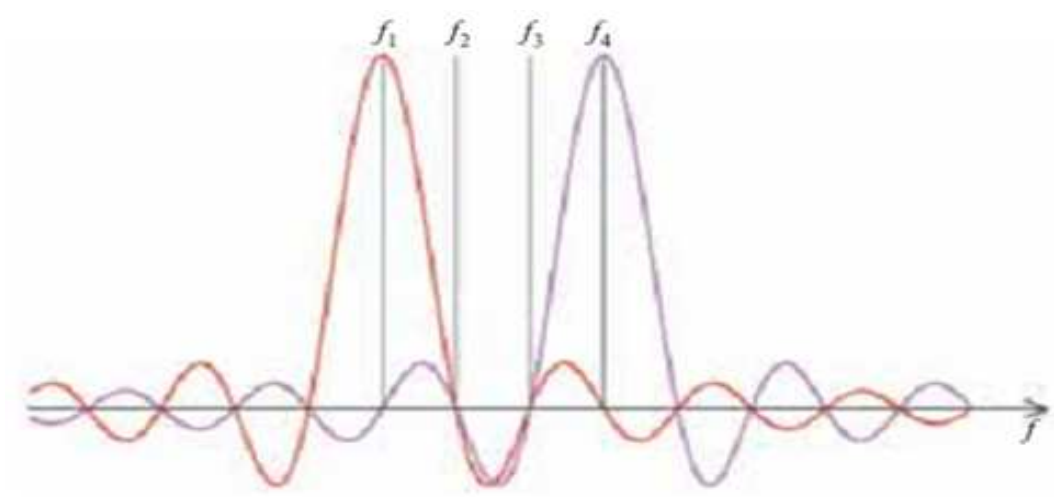

Fig. 9. Spectrum density of the OFDM-symbol with excluded frequencies 


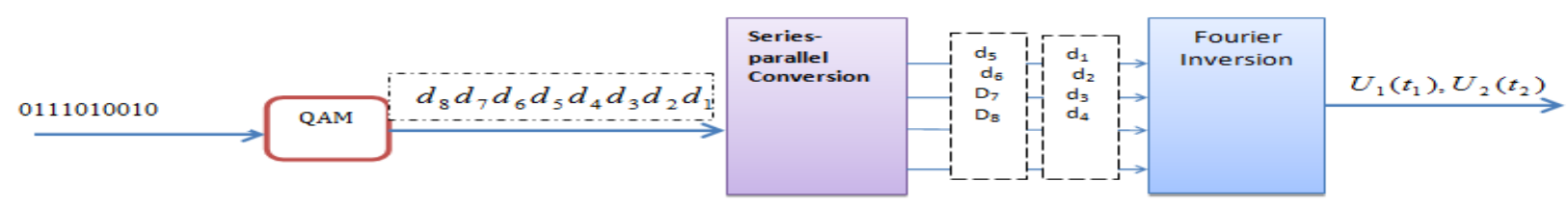

Fig. 10. The block diagram of the OFDM-symbols' of complex envelopes formation with the exception of occupied frequencies

Thus, the total data transfer rate in a micro-cellular network is reduced proportionally to the number of the subcarriers excluded. In order to avoid data loss during subcarriers' reduction to zero the rate of information bit stream at the input of the QAM-modulator should be coordinated with the available frequency resource. According to the model of interaction of open OSI systems, the bit stream rate control challenge is met by the transport layer (Rault et al., 2014).

\section{The Algorithms for Data Transfer in a Micro- Cellular Network}

This process can be implemented using various scenarios of downlink (BS-SS) and the uplink (SS-BS) organization:

- In case of downlink - on the free frequencies of a macro-cellular network, in case of uplink-via GPRS/EDGE

- On the free frequencies for uplink and downlink

When using GPRS/EDGE in case of uplink the traffic asymmetry is taken into account in the direction from/to the subscriber. The advantage of this option is a maximum speed in case of downlink. Disadvantages: Absence or occupancy of GPRS/EDGE-channels excluding the operation of a micro-cellular network; the need to change the operating GSM network in terms of coordinating the work of the GPRS/EDGE subsystem and micro-cellular data transfer network; limited data transfer rates in case of uplink.

The advantages of data transfer on free frequencies in both directions are independence from occupancy or availability of GPRS/EDGE and flexible control of rate in the directions from/to the subscriber.

The disadvantage is a lower downlink rate compared with the rate when using the OFDM only for downlink.

\section{Conclusion}

The proposed Study spectrum sensing in Cognitive Radios secondary the demand for spectrum in wireless environment has grown substantially due to the widespread use of smart phones. We have proposed a cognitive radio which senses and splits the spectrum. Cyclo-stationary spectrum sensing is the best spectrum sensing technique. Principles of cognitive radio are capable of data transfer in parallel with the operation of the main GSM network with the rate of several dozens of megabits per second depending on the configuration of the operating GSM network. The High-speed for Data transmission in GSM networks based on Cognitive Radio (CR) technology and its various aspects that may play a very important role in the field of next generation wireless communication networks.

The future work will focus on enhance the result and simulate look at ways, Cyclo-Stationary detection for over Networks Based on Cognitive Radio, use other modulation and is analyzing and evaluating the sensing characteristics of the new cognitive radio physical layer modulation scheme.

\section{Acknowledgement}

This Work Was Supported by the Deanship of Scientific Research, Jerash University.

\section{Author's Contributions}

All authors contributed equally to the implementation of the present paper.

\section{Ethics}

This article is original and contains unpublished Material, the corresponding author confirms that all of the other authors have read and approved the manuscript and no ethical issues involved.

\section{References}

Mei Akyildiz, I.F., W.Y. Lee and K.R. Chowdhury, 2009. CRAHNs: Cognitive radio ad hoc networks. AD Hoc Netw., 7: 810-836.

DOI: 10.1016/j.adhoc.2009.01.001

Akyildiz, I.F., W.Y. Lee, M.C. Vuran and S. Mohanty, 2006. NeXt generation/dynamic spectrum access/cognitive radio wireless networks: A survey. Comput. Netw., 50: 2127-2159.

DOI: 10.1016/j.comnet.2006.05.001

Anis, M., M. Ortmanns and N. Wehn, 2010. RF spectrum sensing technique for cognitive UWB radio network. Proceedings of the ESSCIRC, IEEE Xplore Press, pp: 506-509.

DOI: 10.1109/ESSCIRC.2010.5619754 
Baldini, G., I.N. Fovino, M. Masera, M. Luise and V. Pellegrini et al., 2010. An early warning system for detecting GSM-R wireless interference in the highspeed railway infrastructure. Int. J. Critical Infrastructure Protect., 3: 140-156. DOI: $10.1016 /$ j.ijcip.2010.10.003

Benedetto, D.M, Y. Hua, T. Kaiser and X. Wang, 2008. Cognitive radio technology. IEEE Signal Process. Magaz., 25: 10-198. DOI: 10.1109/MSP.2008.930499

Chen, D., D. Qu and T. Jiang, 2010. Novel prototype filter design for FBMC based cognitive radio systems through direct optimization of filter coefficients. Proceedings of the International Conference on Wireless Communications and Signal Processing, IEEE Xplore Press, pp: 1-6. DOI: 10.1109/WCSP.2010.5633530

Dashti, M., P. Azmi and K. Navaie, 2012. Resource allocation for underlay CDMA cognitive radio networks. Proceedings of the IEEE Wireless Communications and Networking Conference, Apr. 1-4, IEEE Xplore Press, pp: 2792-2976.

DOI: 10.1109/WCNC.2012.6214276

Ekin, S., M.M. Abdallah, K.A. Qaraqe and E. Serpedin, 2012. Random subcarrier allocation in OFDM-based cognitive radio networks. IEEE Trans. Signal Process., 60: 4758-4774.

DOI: 10.1109/TSP.2012.2203126
Khan, A.A., M.H. Rehmani and M. Reisslein, 2016. Cognitive radio for smart grids: Survey of architectures, spectrum sensing mechanisms and networking protocols. IEEE Commun. Surveys Tutorials, 18: 860-898. DOI: 10.1109/COMST.2015.2481722

Ng, D.W.K., E.S. Lo and R. Schober, 2016. Multiobjective resource allocation for secure communication in cognitive radio networks with wireless information and power transfer. IEEE Trans. Vehicular Technol., 65: 3166-3184. DOI: 10.1109/TVT.2015.2436334

Parvin, S., F.K. Hussain, O.K. Hussain, S. Han and B. Tian et al., 2012. Cognitive radio network security: A survey. J. Netw. Comput. Applic., 35: 1691-1708. DOI: $10.1016 /$ j.jnca.2012.06.006

Rault, T., A. Bouabdallah and Y. Challal, 2014. Energy efficiency in wireless sensor networks: A top-down survey. Comput. Netw., 67: 104-122. DOI: 10.1016/j.comnet.2014.03.027

Stevenson, C.R., G. Chouinard, L. Zhongding, W. Hu and S. Shellhammer et al., 2009. IEEE 802.22: The first cognitive radio wireless regional area network standard. IEEE Commun. Magazine, 47: 130-138. DOI: 10.1109/MCOM.2009.4752688 